correspondants. Voici le résultat au quel j'ai cru pouvoir m'arrêter:

$$
\begin{aligned}
\Omega & =229^{\circ} 9^{\prime} 0(1880.0) \\
\pi--\delta & =7524.2 \\
\mathrm{i} & =11121.7 \\
\mathrm{e} & =0.6989 \\
\mathrm{a} & =2^{\prime \prime} 400 \\
\mathrm{U} & =55^{\mathrm{n}} 582 \\
\mathrm{~T} & =1882.774 .
\end{aligned}
$$

\begin{tabular}{|c|c|c|c|c|c|}
\hline & Observ & ations & Calc & & \\
\hline Epoque & pos. & dist. & pos. & dist. & Observateur \\
\hline 1834.47 & $37^{0} 10$ & - & $+1^{0} 61$ & - & J. Herschel \\
\hline 1835.55 & 36.80 & - & -0.51 & - & 》 \\
\hline 1836.43 & 34.50 & - & +0.06 & - & $\eta$ \\
\hline 1837.21 & $\ldots$ & $2^{\prime \prime} 66$ & - & $-0^{\prime \prime} 39$ & $\pi$ \\
\hline 1837.43 & 32.70 & - & -0.04 & - & 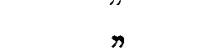 \\
\hline 1847.32 & 14.10 & 2.30 & $t-0.13$ & -0.13 & Jacob" \\
\hline 1850.46 & 5.87 & 2.29 & +1.34 & -0.27 & 7 \\
\hline 1851.54 & 447 & 2.26 & +0.05 & -0.30 & נI \\
\hline 1852.27 & 3.45 & 1.89 & -0.78 & +0.03 & 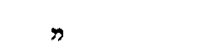 \\
\hline 1852.72 & 0.97 & 1.91 & +0.07 & -0.02 & 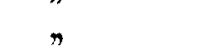 \\
\hline 1853.25 & 359.58 & 1.83 & \pm 0.00 & +0.03 & n \\
\hline 1853.71 & 358.57 & - & $\overline{+} 0.13$ & - & Powell \\
\hline 1853.78 & 358.50 & 1.82 & -0.02 & +0.01 & Jacob \\
\hline 1854.86 & 256.16 & 1.71 & +0.48 & -0.10 & $\eta$ \\
\hline 1854.78 & 355.58 & - & -0.09 & -- & Powell \\
\hline 1855.77 & 352.93 & - & -0.60 & - & $r$ \\
\hline 1856.21 & 350.80 & 1.68 & -0.42 & +0.02 & Jacob \\
\hline 1856.67 & 348.08 & 1.66 & +1.21 & +0.01 & n \\
\hline 1857.21 & 348.44 & 1.67 & -1.06 & -0.02 & , \\
\hline 1857.66 & 346.29 & 1.55 & -0.52 & -0.08 & , \\
\hline 1858.20 & 343.42 & 1.53 & +0.29 & +0.07 & m \\
\hline 1859.72 & 338.10 & - & -0.52 & - & Powell \\
\hline 1861.69 & 328.80 & -- & +0.03 & - & $\rightarrow$ \\
\hline 1862.27 & 325.30 & - & +0.78 & - & , \\
\hline 1863.84 & 318.10 & - & +0.19 & - & , \\
\hline 1875.65 & 257.41 & 1.45 & +0.18 & \pm 0.00 & Schiaparelli \\
\hline
\end{tabular}

La comparaison de ces éléments avec les observations donne le tableau suivant:
On roit que les observations le position sont representées à peu prés entre les limites des crreurs possibles: le même a lieu pour les distances à partir de 1852, quion pourrait mème fairc accorder un peu mieux en diminuant le demi-grandaxe de quelques centièmes de seconde. Pour ce qui regarde les quatre premières distances, il est impossible d'y satisfaire avec un système d'éléments quelconque, sans admettre des erreurs intolérables pour les angles de position correspondants, ou sans renoncer à l'uniforme description des aires: elles sont probablement trop grandes.

J'ajoute une ephéméride pour les années qui vont suivre jusqu'au prochain passage au périhélie en 1882. L'étoile se resserre de plus en plus et les observations, qui pour une déclinaison de $-37^{\circ} 15^{\prime}$ sont déjà si difficiles sous notre latitude, deviendront bientôt impossibles.

$\begin{array}{cccc}\text { Anom. excentr. Date curr. } & \text { Posit. } & \text { Dist. } \\ 275^{\circ} & 1875.811 & 256^{\prime \prime} 65 & 1^{\prime \prime} 45 \\ 280 & 1876.510 & 253.40 & 1.45 \\ 285 & 1877.167 & 250.35 & 1.44 \\ 290 & 1877.777 & 247.45 & 1.42 \\ 295 & 1878.340 & 244.67 & 1.39 \\ 300 & 1878.865 & 241.97 & 1.35 \\ 305 & 1879.346 & 239.32 & 1.31 \\ 310 & 1879.791 & 236.68 & 125 \\ 315 & 1880.198 & 234.02 & 1.18 \\ 320 & 1880.571 & 231.25 & 1.11 \\ 325 & 1880.916 & 228.33 & 1.02 \\ 330 & 1881.230 & 225.10 & 0.93 \\ 335 & 1881.526 & 221.45 & 0.83 \\ 340 & 1881.801 & 217.10 & 0.73 \\ 345 & 1882.059 & 211.57 & 0.62 \\ 350 & 1882.303 & 204.00 & 0.51 \\ 355 & 1882.544 & 192.72 & 0.40 \\ 360 & 1882.774 & 174.52 & 0.31\end{array}$

Observatoire de Brera in Milan, 22. Décembre 1875. J. V. Schiaparelli.

\title{
Beobachtungen der Sonnenflecken zu Athen. 1875.
}

\begin{tabular}{rll} 
Datum & \multicolumn{2}{c}{ Ubr Grupp } \\
Januar 1 & -8.2 & 3 \\
2 & -8.3 & 3 \\
3 & -8.5 & 2 \\
4 & -10.6 & 1 \\
5 & -8.7 & 1 \\
6 & -8.3 & 0
\end{tabular}

\begin{tabular}{rrr|} 
Datum & \multicolumn{2}{c}{ Uhr Grupp. } \\
Januar 7 & -8.3 & 1 \\
8 & -9.0 & 1 \\
9 & -8.3 & 1 \\
10 & -8.2 & 1 \\
11 & -10.1 & 2 \\
12 & -9.2 & 2
\end{tabular}

Datum Januar 13

Uhr Grupp.

$$
\begin{array}{rrr}
13 & 2.3 & 2 \\
14 & -9.4 & 1 \\
15 & -8.2 & 2 \\
16 & -9.8 & 1 \\
17 & -8.6 & 2 \\
18 & -.8 .5 & 1
\end{array}
$$


Datum Uhr Girupp. Datum Uhr Grupp. Januar 26 $\begin{array}{lll}27 & 1.6 & 0\end{array}$ $\begin{array}{lll}28 & 1.6 & 0\end{array}$ $29 \quad 0.0 \quad 0$ $\begin{array}{lll}30 & 0.1 & 0\end{array}$ $31-9.80$

Februar $\begin{array}{lll}4 & 0.1 & 1\end{array}$

j $\quad 0.3 \quad 1$

$\begin{array}{lll}6 & -11.8 & 1\end{array}$ $2.2 \quad 1$ 2.21 2.11

$10-9.1 \quad 0: \therefore$ :

$\begin{array}{lll}11 & -8.3 & 1\end{array}$

$\begin{array}{lll}12 & -8.3 & 1\end{array}$

$13-9.5 \quad 0$ :.: $\begin{array}{ll}2.1 & 0\end{array}$

$15-8.60$

$16-8.80$

$\begin{array}{lll}17 & 1.9 & 0\end{array}$

$\begin{array}{lll}18 & 3.5 & 0\end{array}$

$19 \quad 3.1 \quad 1$ :.:

$20-8.52$

$21 \quad 3.0 \quad 2$

$22-8.32$

$23-8.6 \quad 3$

$24 \quad 2.1 \quad 4$

$25-8.64$

$26-8.3 \quad 5$

$\begin{array}{llll}27 & 2.1 & 4\end{array}$

$\begin{array}{llll}28 & \ldots . . & 8.5 & 3\end{array}$

März $\quad 1-8.5 \quad 4$

$2 \quad 2.1 \quad 2$ :.:

$3-8.32$
4

$\begin{array}{lll}4 & -8.3 \quad 3\end{array}$

$5-8.43$

$\begin{array}{llll}6 & -10.1 & 1\end{array}$

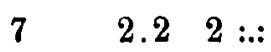

$8-8.23$

$9-8.23$

$\begin{array}{lll}10 & -7.9 & 3\end{array}$

$11-8.31$

$\begin{array}{lll}12 & 1.6 & 2\end{array}$

$13-8.22$

$14-8.92$

$16 \quad 2.3 \quad 2: .:$

$\begin{array}{lll}17 & 2.4 & 4\end{array}$

$18-8.5 \quad 5$
März $20-82 \quad$

$$
\begin{array}{rrl}
21 & 2.3 & 2 \\
22 & -9.9 & 2 \text { :.: }
\end{array}
$$

$23-8.52$

$\begin{array}{lll}24 & -7.9 & 2\end{array}$

$\begin{array}{lll}25 & -7.9 & 2\end{array}$

$\begin{array}{llll}26 & --8.3 & 2\end{array}$

$\begin{array}{lll}27 & 1.8 & 1\end{array}$

$\begin{array}{llll}28 & -7.9 & 1\end{array}$

$29-8.3 \quad 3$

$31-8.63$ :.:

$2-8.53$

$3-8.24$

$4-8.13$

$\begin{array}{lll}6 & 1.9 & 1\end{array}$

$\begin{array}{lll}7 & -8.5 & 1\end{array}$

$\begin{array}{llll}8 & 2.3 & 1 & \therefore:\end{array}$

$9-8.20$

$10-8.20$

$11-7.81$

$\begin{array}{lll}12 & -7.9 & 2\end{array}$

$13-7.72$

$14-7.8 \quad 2$

$\begin{array}{lll}15 & 2.5 & 2\end{array}$

$16-7.63$

$17-7.7 \quad 3$

$18-7.9 \quad 3$

$\begin{array}{llll}19 & --8.1 & 2\end{array}$

$\begin{array}{llll}20 & -7.8 & 2\end{array}$

$\begin{array}{llll}21 & -7.7 & 1\end{array}$

$22-7.6 \mathrm{l}$

$23-7.8 \quad 1$

$24-7.9 \quad 1$

$25-8.12$

$26-7.91$

$27-7.92: .$.

$\begin{array}{llll}28 & --7.9 & 2\end{array}$

$29 \quad 2.1 \quad 2$ :.:

$30-8.13$

$\begin{array}{llll}\mathrm{Mai} & 1 & -7.8 \quad 3\end{array}$

$2-7.83$

$3-7.9 \quad 3$

$\begin{array}{lll}4 & -7.9 \quad 2\end{array}$

$\begin{array}{lll}5 & -10.3 & 2\end{array}$

$6-7.81$

$\begin{array}{lll}7 & -7.4 & 1\end{array}$

$8-7.61$
April $1 \quad 3.13$ :.:

$5-8.62: .:$
Datım Uhr Grupp.

Mai $9-7.60$

$\begin{array}{rrr}10 & 2.10 \\ 11 & -7.6 & 0\end{array}$

$12-7.50$

$\begin{array}{lll}13 & -.10 .7 & 0\end{array}$

$14-7.50$

$15-7.60$

$16-7.90$

$17-7.70$

$18-7.80$

$19-7.80$

$20-7.90$

$21-7.50$

$22-7.80$

$\begin{array}{lll}23 & -7.7 & 0\end{array}$

$\begin{array}{lll}24 & 2.5 & 2\end{array}$

$25-7.6 \quad 3$

$26-8.12$

$\begin{array}{llll}27 & -7.7 & 1\end{array}$

$\begin{array}{lll}28 & -7.6 & 1\end{array}$

$\begin{array}{llll}29 & -7.8 \quad 1\end{array}$

$\begin{array}{llll}30 & 2.2 & 1 & : .\end{array}$

$31-7.91$

$\begin{array}{llll}\text { Juni } & 1 & -7.9 & 2\end{array}$

$2-7.82$

$\begin{array}{llll}3 & -8.9 & 1\end{array}$

$4-7.81$
5.7

$5^{* *}-7.72$

$6-7.92$

$\begin{array}{lll}7 & -7.9 & 1\end{array}$

$8-7.81$

$\begin{array}{lll}9 & -7.9 & 1\end{array}$

$10-7.8 \quad 1$

$11-7.81$

$12-8.10$

$13-7.80$

$14-8.0 \quad 0$

$\begin{array}{lll}15 & -7.8 & 0\end{array}$

$\begin{array}{lll}16 & -7.7 \quad 2\end{array}$

$\begin{array}{lll}17 & -7.6 \quad 1\end{array}$

$\begin{array}{lll}18 & -8.1 & 1\end{array}$

$\begin{array}{lll}19 & -7.9 & 1\end{array}$

$20-7.81$

$\begin{array}{llll}21 & -7.8 & 1\end{array}$

$22-8.12$

$23-7.82$

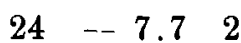

$25-7.81$

$\begin{array}{lll}26 & -7.8 & 1\end{array}$
Datum Uhr Grupp.

Juni $27-8.12$

$28-7.8 \quad 2$

$\begin{array}{lll}29 & -7.5 \quad 2\end{array}$

$30-7.92$

Juli $\quad \begin{array}{lll} & -7.7 & 2\end{array}$

$2 * * \quad 2.0 \quad 1$

$\begin{array}{lll}3 & -7.7 & 1\end{array}$

$\begin{array}{lll}4 & -7.8 & 1\end{array}$

$5-7.71$

$\begin{array}{lll}6 & -7.7 & 1\end{array}$

$\begin{array}{lll}7 & -7.6 & 1\end{array}$

$\begin{array}{lll}8 & -7.7 & 1\end{array}$

$\begin{array}{llll}9 & -8.1 & 1\end{array}$

$\begin{array}{lll}10 & -7.8 & 1\end{array}$

$\begin{array}{lll}11 & -7.6 & 1\end{array}$

$\begin{array}{lll}12 & -7.8 & 1\end{array}$

$\begin{array}{lll}13 & -7.9 & 1\end{array}$

$\begin{array}{lll}14 & -7.9 & 1\end{array}$

$15-7.6 \quad 1$

$\begin{array}{lll}16 & -7.8 & 0\end{array}$

$17-7.61$

$\begin{array}{lll}18 & -7.6 & 1\end{array}$

$\begin{array}{lll}19 & -7.9 & 0\end{array}$

$20-7.90$

$21 \quad-7.8 \quad 0$

$22-7.60$

$23-7.70$

$24-7.80$

$25 \quad-7.7 \quad 0$

$\begin{array}{lll}26 & -7.8 & 0\end{array}$

$\begin{array}{llll}27 & -7.6 & 1\end{array}$

$28-7.61$

$\begin{array}{lll}29 & -7.7 & 1\end{array}$

$30-7.81$

$31-7.81$

August $1^{*}-8.51$

$2-7.82$

$\begin{array}{lll}3 & -7.9 & 2\end{array}$

$4-7.92$
5

$5-6.82$

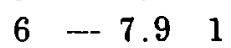

$\begin{array}{lll}7 & -8.0 & 0\end{array}$

$8-8.10$

$9-8.0 \quad 0$

$10-7.90$

$11-7.7 \quad 0$

$12-7.80$

$\begin{array}{lll}13 & -7.9 & 0\end{array}$

$14-8.10$ 
Datum Uhr Grupp.

$\begin{array}{rrr}\text { August 15 } & -7.8 & 0 \\ 16 & -7.8 & 0 \\ 17 & -8.1 & 0 \\ 18 & -7.9 & 0 \\ 19 & -7.8 & 0 \\ 20 & -8.1 & 0 \\ 21 & -7.8 & 1 \\ 22^{* 5} & -7.7 & 2 \\ 23 & -7.7 & 2 \\ 24 & -7.7 & 2 \\ 25 & -7.8 & 2 \\ 26 & -7.7 & 2 \\ 27 & -7.7 & 2 \\ 28 & -7.9 & 2 \\ 29 & -7.9 & 1 \\ 30 & -7.8 & 1 \\ 31 & -7.9 & 1\end{array}$

Septbr. $1-7.91$

$2-7.7 \quad 0$

$3-7.90$

$4-8.10$

$5 *-8.70$

$\begin{array}{lll}6 & -7.7 & 0\end{array}$

$7-7.80$

$8-7.90$

$9-7.90$

$\begin{array}{lll}10 & -7.9 & 1\end{array}$

$\begin{array}{lll}11 & -8.0 \quad 1\end{array}$

$12 *-8.80$

$13-7.9 \quad 0$

$14-7.90$.

$15-7.81$

$16-8.11$

$17-7.8 \quad 0$

$18-8.10$

$19^{*}-7.8 \quad 0$

$20-7.90$

$21-7.80$

$22-7.80$

$23-7.80$

$24-7.90$

$\begin{array}{llll}25 & -8.1 & 0\end{array}$

$26 *-11.0 \quad 1$
Datum Uhr Grupp.

Sept. $27 \quad-7.8 \quad 1$

$28-7.9 \quad 1$

$\begin{array}{lll}29 & -7.9 & 2\end{array}$

$\begin{array}{lll}30 & -8.1 & 2\end{array}$

October $2-7.82$

$\begin{array}{lll}3 & -8.1 & 2\end{array}$

$4-7.8 \quad 2$
5

$\begin{array}{llll}5 & -7.8 & 2\end{array}$

$\begin{array}{lll}6 & -7.9 & 1\end{array}$

$\begin{array}{lll}7 & -7.82\end{array}$

$\begin{array}{lll}8 & -8.3 \quad 1\end{array}$

$\begin{array}{lll}9 & -7.7 & 2\end{array}$

$10-8.0 \quad 0$

$11-7.90$

$12-8.10$

$13-7.90$

$14-7.9 \quad 0$

$\begin{array}{lll}15 & -10.3 & 0\end{array}$

$16-7.80$

$17-7.80$

$18-7.80$

$\begin{array}{lll}19 & -8.2 & 0\end{array}$

$\begin{array}{lll}20 & -7.9 & 1\end{array}$

$\begin{array}{llll}21 & -7.7 & 1\end{array}$

$22-7.81$

$23-7.81$

$24-8.41$

$25-7.92$

$\begin{array}{lll}26 & -7.9 & 1\end{array}$

$\begin{array}{lll}27 & -7.9 & 2\end{array}$

$28-7.92$

$29 * \quad 1.4 \quad 1$

$\begin{array}{lll}30 & -7.9 & 1\end{array}$

$\begin{array}{llll}31 & -7.9 & 1\end{array}$

Novbr. $1-8.71$

$\begin{array}{llll}2 & --11.5 & 1\end{array}$

$\begin{array}{lll}3 & -8.8 & 2\end{array}$

$\begin{array}{llll}5 & -7.9 & 1\end{array}$

$6-8.10$

$7-8.10$

$8-8.10$

$\begin{array}{llll}9 & -8.1 & 0\end{array}$

$10-8.0 \quad 0$
Datum Uhr Grupp.

Novbr.11 - 7.90

$12-8.80$

$13-8.10$

$14-7.9^{\circ} 0$

$\begin{array}{llll}15 & -\ldots & 7.8 & 0\end{array}$

$16^{* *}-8.10$

$17 * \quad 1.2 \quad 0$

$18^{* *}--11.51$

$19-11.2 \quad 2$

$20-8.13$

$\begin{array}{llll}21 & -8.0 & 8.0 & 4\end{array}$

$22-8.14$

$23^{* *}-9.54$

$24-8.1 \quad 5$

$25 \quad-.7 .95$

$\begin{array}{lll}26 & -8.2 & 4\end{array}$

$27-8.0 \quad 3$

$28 * *-10.0 \quad 1$

$\begin{array}{llll}29 & - & -8.0 & 1\end{array}$

$\begin{array}{lll}30 & -10.6 & 1\end{array}$

Decbr. $1^{* * \ldots} 7.90$

$2-8.10$

$3 * *-9.3 \quad 0$

$\begin{array}{lll}4 & -10.5 & 0\end{array}$

Datum Uhr Grupp.

Decbr. $5-8.70$

$6-7.90$

7
$7-7.9 \quad 0$

$8-8.80$

$9-8.30$

$10-7.90$

$\begin{array}{lll}12 & -8.4 \quad 2\end{array}$

$\begin{array}{lll}13 & -8.7 & 2\end{array}$

$14-8.02$

$\begin{array}{llll}15 & -8.2 & 1\end{array}$

$\begin{array}{lll}16 & -8.7 & 1\end{array}$

$\begin{array}{llll}17 & - & -8.2 & 2\end{array}$

$\begin{array}{lll}18 & -8.3 & 2\end{array}$

$20-9.02$

$21-8.22$

22
$-8.9 \quad 1$

$23-8.31$

$\begin{array}{llll}24 & -8.2 \quad & \text { l }\end{array}$

$25^{* *} .2 .5 \quad 1$

$26 * \quad 1.6 \quad 1$

$27 * \quad 4.3 \quad 1$

$29 * *-8.3 \quad 1$

$30 * \quad 1.0 \quad 1$

$\begin{array}{lll}31 & -9.6 & 0\end{array}$

Die Zäblung dow Gruppen on Donmennecken ward im Jahr 1875 rom Anfang Januar bis Juni 3 ron meinen beiden Gehülfen besorgt, und zwar an einem kleinen Refractor von etwa 25maliger Vergrösserung. Nach Juni 3 habe ich $9 \mathrm{Mal}$ allein, und $12 \mathrm{Mal}$ gemeinschaftlich mit dem ältern Gehülfen beobachtet. Es zählte die Gruppen Herr Alex. Wurlisch: Januar 1 bis 16, Februar 7, Februar 14 bis Juni 2, sodann Juni 4 bis zu Ende des Jahrs. Der jüngere Gehülfe Johannes Chatzidakis beobachtete Januar 17 bis Februar 6, Februar 8 bis 13 und Juni 3; und da es seine ersten Versuche waren, mögen ihm gelegentlich sehr feine Gruppen entgangen sein. Wo ich allein beobachtete, findet man im Verzeichniss einen *; dagegen bedeuten 2 solche Sterne (**), dass ich gemeinschaftlich mit dem Gehülfen nach Flecken suchte. An 10 Tagen haben Wolken die Beobachtung verhindert.

Athen, den 1. Januar 1876.

J. F. Julius Schmidt. 